Voix et Images

voixetimages

\title{
De la transparence à l'opacité. Quelques formes du lisible
}

\section{Frances Fortier}

Volume 32, numéro 3 (96), printemps 2007

Le dix-neuvième siècle québécois et ses modèles européens

URI : https://id.erudit.org/iderudit/016582ar

DOI : https://doi.org/10.7202/016582ar

Aller au sommaire du numéro

\section{Éditeur(s)}

Université du Québec à Montréal

\section{ISSN}

0318-9201 (imprimé)

1705-933X (numérique)

Découvrir la revue

Citer ce compte rendu

Fortier, F. (2007). Compte rendu de [De la transparence à l'opacité. Quelques formes du lisible]. Voix et Images, 32(3), 127-131.

https://doi.org/10.7202/016582ar d'utilisation que vous pouvez consulter en ligne.

https://apropos.erudit.org/fr/usagers/politique-dutilisation/ 


\title{
R O M A N
}

\author{
De la transparence à l'opacité. \\ Quelques formes du lisible \\ $++$
}

FRANCES FORTIER

Université du Québec à Rimouski

Que peuvent avoir en commun un récit de filiation qui hurle des pulsions primaires, un faux journal qui se construit sur une médiation intertextuelle et un texte résolument «barbare » qui oblige à lire la lettre du langage? Rien, sinon qu'ils permettent de cartographier quelques enjeux pragmatiques du roman d'aujourd'hui, qu'on croit généralement emmuré dans un narcissisme exaspéré par des décennies de pratiques autobiographiques. Si les stratégies déployées sont absolument singulières, elles témoignent néanmoins, parfois de manière oblique, d'un souci du lecteur construit à même des écrans de lisibilité plus ou moins opaques, qu'il s'agisse de le provoquer, de faire appel à son savoir ou à sa connivence. Sollicité par le biais d'un langage transitif, qui laisse croire à une confession intime, le lecteur s'abandonnera volontiers à la libido sciendi ; happé par un personnage déjà façonné par la littérature, il se fera herméneute et tentera d'en saisir les plus fines déclinaisons; abasourdi par un langage qui cultive à première vue l'illisibilité, il tentera d'entrer dans le jeu et de construire une voie d'accès. Les trois romans retenus explorent ces régimes de lecture, avec audace et maîtrise. Qu'on en juge plutôt.

«Je suis une fille de pute» (11) précise d'entrée de jeu la narratrice de Crève, maman! ${ }^{1}$ de Mô Singh, assumant du coup l'invective du titre et tentant de la justifier. Un procès haineux va s'enclencher, alors que la mère gît à l'hôpital dans un coma irréversible après une sixième tentative de suicide et que sa fille, à son chevet, souhaite sa mort en se remémorant tous les sévices subis. Ce récit de filiation qui inverse l'enjeu de mémoire remonte à la naissance de la mère détestée, elle aussi née d'une "petite pute indienne» (23) jamais revue et victime d'abus répétés dès l'âge de huit ans. Toute la tension de l'écriture réside dans ce refus de l'héritage, dans cette détestation de la mère qui est aussi haine de soi, dans ce désir de faire dévier sa propre trajectoire, de s'extirper soi-même d'un destin de violence.

$$
+++
$$

1 Mô Singh, Crève, maman!, Montréal, XYZ éditeur, 2006, 144 p. 
Fragmenté en scènes d'une page ou deux, le récit cumule les faits bruts, entrecoupe la réminiscence des viols de l'enfance de la description des jeux érotiques de l'adulte - «Depuis onze ans, je suis la femme d'un étalon» (14) - qui laisse poindre le désarroi d'une identité à la fois admise et refusée. Ainsi, alors que la narratrice est au chevet de sa mère, ledit compagnon réserve une chambre de motel minable à côté de l'hôpital où elle court le rejoindre:

Il veut mon cul et j'ai envie de le lui donner.

Manu, c'est un spécialiste de l'expérimentation sexuelle. Il est adepte de l'amour charnel sous toutes ses formes. Chaque fois que nos corps se rencontrent, il tente de me pénétrer, avec ses yeux, avec ses mains, avec sa bouche, avec sa queue, mais ce qu'il préfère, c'est mon cul. Je ne lui offre pas souvent. Chaque fois, je crie. Je suis couchée sur le ventre et mes fesses se crispent. Je le prive de son plaisir le temps d'une brève réflexion. J'en ai envie, mais je tremble à l'idée de la douleur. Je demeure sur mes positions. Son sexe respire près de l'endroit interdit. Je lui interdis de bouger. Je prendrai l'initiative. Je le ferai entrer millimètre par millimètre jusqu'à ce qu'il éjacule, avant même qu'il n'ait atteint toute ma profondeur.

Mon corps perd sa lucidité avec Manu. Il est mon libérateur. (63)

Pure écriture du corps, du corps qui jouit comme du corps qui souffre, sans échappée vers l'imaginaire, sans fantasme, sans intertextualité, Crève maman! joue d'une langue crue et cruelle qui fait de la vie une sorte de loterie où la sexualité est toujours plus ou moins liée à la violence. La narratrice et ses cinq frères et sœurs nés bâtards de pères différents mais tous aussi minables les uns que les autres - «Maman n'a pas choisi les pères de ses enfants. Ils sont tombés dans son lit. Les enfants sont venus après!» (42) - auront vécu un véritable enfer, un ballet des horreurs qui conjugue abus physiques, violence psychologique, coups et blessures. La précision des détails des sévices de cette enfance sordide fait sourdre la question du témoignage, en dépit de la mention générique «roman»: le viol répété par le frère (91), le «coup de poing bien profond dans le ventre (117) donné par la mère, le père retrouvé après des années et qui «insiste pour que j'ouvre mes jambes» (50), le raffinement des punitions corporelles justifient certes, sur le plan de la logique du récit, le cri de haine sauvage lancé à la mère désormais inatteignable dans sa posture de gisante, mais suscitent en même temps un acte de lecture ambigu, oscillant entre le voyeurisme, l'apitoiement et l'indifférence, faisant du lecteur le jouet captif d'une violence reconduite par l'écriture, à l'image de cette narratrice qui attend en silence la mort de sa mère «tout en faisant semblant de souffrir de la voir ainsi immobile» (21).

Les restes de Muriel $^{2}$, le roman de Patrick Boulanger, construit différemment son lecteur, même s'il convoque aussi au passage un univers de violence familiale et

$$
+++
$$

2 Patrick Boulanger, Les restes de Muriel, Montréal, Triptyque, 2007, 96 p. 
sexuelle. Dès la première page, le texte installe son appartenance à la littérature en liant le «départ» de ladite Muriel à une citation de Claude Gauvreau extraite d'une «brique rouge» saisie au hasard dans la bibliothèque et ouverte à «la page quatre cent vingt: "Les supplices chinois, les tortures des Inquisitions: rien de cela ne pouvait être plus intolérable que de supporter qu'elle ne soit pas là." » (9) Le lecteur est prévenu, on rejouera ici Beauté baroque (1966). Il ne s'agira pas pour autant d'enfermer le lecteur dans un canevas intertextuel, mais plutôt de multiplier les entrées dans ce récit extrêmement maîtrisé, qui annonce ses couleurs: "Je suis tombé dans la fiction, je me suis fait personnage, anti-héros d'une œuvre tragicomique burlesque et baroque; je me suis fait pastiche, comme les énormes seins de Marie-Jolie qui m’ont raccompagné à la porte.» (23) Métafiction critique, sans doute, mais constamment déportée vers des enjeux contemporains, qui déroule en parallèle une histoire inextricablement enchevêtrée à celle de Gauvreau.

Le protagoniste Marc décide de "griffonner dans un carnet» (19) les souvenirs qui lui reviennent de Muriel; ce journal, intitulé Les restes de Muriel (42), fait alterner le récit de la déchéance progressive du narrateur et celui de la vie de Muriel. En une suite discontinue de paragraphes séparés par des astérisques, l'histoire s'élabore à coup de fragments allusifs, revenant sans cesse en circonvolutions à la date appréhendée du 2 juillet: «Il y a maintenant sept semaines que Muriel est disparue et il approche, le deuxième jour de juillet, ce terrible jour qui aurait pu être l'apothéose de mon existence n'eut été des choix de Muriel.» (25) Autour de Marc évoluent des personnages «réels» - Marie-Jolie, Maud, Michel, Maurice, Martin et sa femme Marilyn, Marianne, Mathilde (on aura noté l'initiale commune) - et des personnages peints sur le mur, Marcellus premier et Marcellus second, avec lesquels il se bat constamment. La tension narrative est savamment entretenue par l'aller-retour entre la réalité et la littérature, entre le rêve et la perception, entre l'imaginaire et la douleur, entre la mémoire et l'oubli, entre le vrai et le faux, avant de culminer en une révélation finale : «Le 11 mai dernier, [...] lorsque j'ai frappé Muriel au visage et au ventre pour je ne sais quelle raison, elle s'est assurée que ça ne se reproduise plus./Ni sur elle ni sur son fils.» (95-96) Elle s'est pendue alors qu'elle était enceinte d'un peu plus de sept mois d'un enfant à naître le 2 juillet. Au gré de stratégies autoréflexives, de délires intertextuels, de paralipses et de relectures théoriques, le roman de Patrick Boulanger convoque la méditation littéraire pour mettre en scène la violence, qu'elle soit misogyne, castrante, tournée contre soi ou l'autre. Lestée du poids de la bibliothèque, la lecture hésite, à la manière du narrateur qui n'arrive pas à trancher entre le meurtre ou le suicide, et se réjouit, avec lui, de pouvoir se réfugier dans l'espace de la littérature: «Quand j'écris, c'est la fiesta!» (96)

Dans un tout autre registre, Morts de Low Bat ${ }^{3}$ de Patrick Poulin s'ouvre sur un «Fore» qui prévient obliquement le lecteur du «pauperum » qu'il va recevoir en plein

$$
+++
$$

3 Patrick Poulin, Morts de Low Bat, Montréal, Le Quartanier, 2006, 148 p. 
front. Ce qui est mis à mal ici, avec une dextérité savamment déployée et un aplomb remarquable, c'est précisément et tout ensemble, le langage, la littérature, la représentation, les personnages, la narrativité, et encore bien d'autres choses. Pour décrire l'exercice, une piste de départ peut être celle de la définition en abyme de «pauperum» présentée dans le texte:

PAUPERUM: «Mockey écrivit de guingois un textitos-mex fort barbare, mais il s'agissait en même temps d'une formidable table rase.» (W.B.) Cosme transmédiatique soulageant le fondement salarié du socius. Cf. Biblia pauperum. (Jason Armutt joue ainsi pour Nashville, Sean Erfahrung joue ainsi pour Dallas.) (82)

Cette définition pourrait servir de fil d'Ariane dans le dédale textuel proposé puisque, à la manière de cet ensemble de manuscrits et $\mathrm{d}$ 'incunables des quatorzième et quinzième siècles qui reconfiguraient, par les images et les citations de divers auteurs bibliques, la relation entre l'Ancien et le Nouveau Testament, la prose jubilatoire à l'œuvre ici conjugue tant la marge que le canon à la faveur, par exemple, d'un Bonhomme BBQ issu à la fois de la scénographie virtuelle du jeu vidéo et du Quart Livre rabelaisien (1552), rebaptisé pour l'occasion en «Le quart-de-livre selon les mensurations de Gros Luc» (85). On croirait à une bravade de potache, si ce n'était de l'envergure de la visée qui, systématiquement, déconstruit tous les codes de lecture. Voyons un peu.

Une galerie d'une centaine de personnages aux noms farfelus - Comedor Coma, le commandant Langis, D. Bay, Short Dubin, Daffy Taffy, Bod Boni, Ricky Hachey, Bad Bat Oil, Lobe, Rhée, Jos d'Off, etc. - apparaissent au gré du texte, sans pour autant entrer en interaction ou participer à une quelconque intrigue. «Sorte de forme-personne dépourvue de face» (108), le personnage "accomplit des actes purs, mais sans la consistance d'une forme» (116). Roussel vient à l'esprit, un Roussel déjanté il va sans dire, d'autant plus que ces personnages s'auto-engendrent et se métamorphosent constamment, purs produits de langage:

Toutes les têtes font: «[...] Richy le Blais détache le soulier de Paul, lequel entre de plain-pied dans la colère; Richy de Pom alors au rolling lèche son propre visage; Richy de Pom se promène soudain dans le sac de Gilles au magasinage dans les seins de Gilles; Dany dit oui; Wot Jess oblige une douzaine d'enfants à parader en laisse avec des perruques: masque de cuir; le crâne de Richy le bègue se roule en boule dans la grotte obscure; par involution, sa tête s'enroule en coquille d'escargot, pour ne plus sortir, tout comme ses dictionnaires d'ancien, et sa queue qui éjacule autonome: Richy l'Ancien tonne de son dicton: "Revenir"; Otron l'os fume une cigarette; Rotan coupe Richy en deux : Paul et Pom; [...].» (119)

L'espace-temps est lui aussi désinvesti: l'imaginaire mythique de l'odyssée homérique se mue ici en une traversée des Amériques en autocar - «Et Doritos au car de tour. Ce fut Texarcana » (46), «Ce fut Nashville» (47), «Ce fut Baltimore» (55), «Ce fut Mazunte» (58), "Ce fut El Paso» (60) - synthétisée en un "home run homérique filé» (130). Puisant à mille sources, l'interdiscursivité débridée 
désarticule elle aussi toute cohérence: si on peut imaginer un temps que chacun des chapitres se construise sur un substrat textuel qui irait de l'hermétisme mallarméen à la description néoromanesque avec un clin d'œil à Georges Perec par les dialogues entre les «carrés-zoologisme» (143), il faut vite convenir que là n'est pas l'essentiel, tant le texte intègre d'allusions à la culture ambiante, du «Supermarché PetroCanada au club vidéo Nationale Banque» (96), en passant par "le chum de Mike Bedaine, en bousine hickory fumée et en bobettes léopard string» (97) et «la jaquette de Chose avec un monome mauve» (93). Époustouflant. Que reste-t-il, au final, de cette dissolution du narratif? Un magistral exercice de langage, amplifié en deux "Ensemble de grammes invertébrés», aux chapitres deux et six, qui proposent un lexique proprement délirant des néologismes du texte présentés dans l'ordre de leur apparition et non en ordre alphabétique. Bref, voilà une "fiction " qui exige un nouveau pacte de lecture, qui saura faire fi de l'exigence de lisibilité et jouer de connivence: une «Opt. Spéciale» est d'ailleurs offerte au tout dernier paragraphe, alors que "pour sortir sans trace laisser ni perdre sang» (146), on peut laisser un message à cette adresse : lowbat.lobeat@gmail.com.

Le plaisir de lire est fugitif, évanescent, et bien malin qui saurait le contraindre. Tous les textes construisent pourtant leur lecteur, à même les détours du langage, les jeux de structure ou les conventions génériques. Ce lecteur idéal, tout en souplesse, s'abreuve à de multiples sources, au gré de ses émerveillements comme de ses dégoûts; désinhibé, il savoure des spectacles autrement insoutenables; parfois capricieux, il reconnaît mal les subtilités esthétiques ou feint de les ignorer; souvent savant, il jouit d'élucider les énigmes ou d'en légiférer le sens; rarement frivole, il échafaude patiemment, page après page, une bibliothèque imaginaire qui lui fait rempart contre les assauts de la vie. Pur effet textuel, il n'en demeure pas moins le pôle interactif de l'acte littéraire, l'instance interprétante, et l'objet ultime de la séduction. Le pur cri chez Mô Singh, le marquage intertextuel chez Boulanger et le «ballon d'auctor autoritas» (52) à la Poulin ont, indéniablement, atteint leur cible. 